\title{
QUANTUM NP AND A QUANTUM HIERARCHY
}

\author{
(Extended Abstract)
}

\author{
Tomoyuki Yamakami \\ School of Information Technology and Engineering \\ University of Ottawa, Ottawa, Ontario, Canada K1N 6N5 \\ yamakami@site.uottawa.ca
}

\begin{abstract}
The complexity class NP is quintessential and ubiquitous in theoretical computer science. Two different approaches have been made to define "Quantum NP," the quantum analogue of NP: NQP by Adleman, DeMarrais, and Huang, and QMA by Knill, Kitaev, and Watrous. From an operator point of view, NP can be viewed as the result of the $\exists$-operator applied to P. Recently, Green, Homer, Moore, and Pollett proposed its quantum version, called the $\mathrm{N}$-operator, which is an abstraction of NQP. This paper introduces the $\exists^{\mathcal{Q}}$-operator, which is an abstraction of QMA, and its complement, the $\forall^{\mathbf{Q}}$-operator. These operators not only define Quantum NP but also build a quantum hierarchy, similar to the Meyer-Stockmeyer polynomial hierarchy, based on two-sided bounded-error quantum computation.
\end{abstract}

Keywords: quantum quantifier, quantum operator, quantum polynomial hierarchy

\section{What is Quantum NP?}

Computational complexity theory based on a Turing machine (TM, for short) was formulated in the 1960s. The complexity class NP was later introduced as the collection of sets that are recognized by nondeterministic TMs in polynomial time. By the earlier work of Cook, Levin, and Karp, NP was quickly identified as a central notion in complexity theory by means of NP-completeness. NP has since then exhibited its rich structure and is proven to be vital to many fields of theoretical computer science. Meyer and Stockmeyer [13] further extended NP into a hierarchy, known as the polynomial (time) hierarchy. This hierarchy has inspired many tools and techniques, e.g., circuit lower-bound proofs and micro hierarchies within NP. There is known to be a relativized world where 
the hierarchy forms an infinite hierarchy. It is thus natural to consider a quantum analogue of NP, dubbed as "Quantum NP," and its extension. Several approaches have been made over the years to define Quantum NP.

As is known, NP can be characterized in several different manners. As the first example, NP can be characterized by probabilistic TMs with positive acceptance probability. Adleman et al. [1] introduced the complexity class NQP as a quantum extension of this probabilistic characterization. Subsequently, NQP (even with arbitrary complex amplitudes) was shown to coincide with the classical counting class co- $\mathrm{C}_{=} \mathrm{P}[7,6,20]$. This shows the power of quantum computation.

NP can be also characterized by logical quantifiers over classical (binary) strings of polynomial length. This is also known as the "guess-and-check" process. Knill [10], Kitaev [9], and Watrous [16] studied the complexity class QMA (named by Watrous), which can be viewed as a quantum extension of the aforementioned quantifier characterization of NP. In their definition, a quantifier bounds a quantum state instead of a classical string. We call such a quantifier a quantum quantifier to emphasize the scope of the quantifier being quantum states. Using this terminology, any set in QMA is defined with the use of a single quantum quantifier over polynomial-size quantum states. It appears that a quantum quantifier behaves in quite a distinctive manner. For instance, Kobayashi et al. [12] recently pointed out that allowing multiple quantum quantifiers may increase the complexity of QMA due to quantum entanglement (in [12], QMA $(k)$ is defined with $k$ quantum quantifiers).

From a different aspect, we can view the process of defining NP as an application of an operator that transforms a class $\mathcal{C}$ to another class $\mathcal{D}$. For example, we write co-C to denote the class $\{A \mid \bar{A} \in \mathcal{C}\}$, where $\bar{A}$ is the complement of $A$. This prefix "co" in co- $\mathcal{C}$ can be considered as the complementation operator that builds co- $\mathcal{C}$ from $\mathcal{C}$. Other examples are Schöning's BP-operator [14] and Wagner's C-operator [15]. The classical existential quantifier naturally induces the so-called $\exists$-operator. With this $\exists$-operator, NP is defined as $\exists \cdot P$. Similarly, we can consider a quantum analogue of the $\exists$-operator. One possible analogue was recently proposed by Green et al. [8]. They introduced the N-operator, which is an abstraction of NQP.

To make the most of quantum nature, we define in this paper a quantum operator that expands the quantum existential quantifier used for QMA and $\mathrm{QMA}(k)$. This quantum operator is called the $\exists^{\mathrm{Q}}$-operator (whose complement is the $\forall^{Q}$-operators). These quantum operators give a new definition for Quantum NP and its expansion, a quantum analogue of the polynomial hierarchy. Our quantum operators, however, require a more general framework than the existing one. In the subsequent section, we discuss a general framework for the quantum operators. 


\section{Toward a General Framework for Quantum Operators}

Let our alphabet $\Sigma$ be $\{0,1\}$ throughout this paper. Let $\mathbb{N}$ be the set of all nonnegative integers and set $\mathbb{N}^{+}=\mathbb{N}-\{0\}$. To describe a quantum state, we use Dirac's ket notation $|\phi\rangle$. Write $\mathcal{H}_{n}$ to denote a Hilbert space of dimension $n$. In comparison with a classical (binary) string, we use the terminology, a quantum string (qustring, for short) of size $n$, to mean a unit-norm vector in $\mathcal{H}_{2^{n}}$. For such a qustring $|\phi\rangle, \ell(|\phi\rangle)$ denotes the size of $|\phi\rangle$. We use the notation $\Phi_{n}$ for each $n \in \mathbb{N}$ to denote the collection of all qustrings of size $n$ and thus, $\Phi_{n} \subseteq \mathcal{H}_{2^{n}}$. Let $\Phi_{\infty}=\bigcup_{n \geq 0} \Phi_{n}$, the set of all finite-size qustrings.

We use a multi-tape quantum Turing machine (QTM), defined in $[4,17]$, as a mathematical model of quantum computations. A multi-tape QTM is equipped with two-way infinite tapes, tape heads, and a finite-control unit. We assume in this paper the following technical restriction on each QTM: a QTM is always designed so that all computation paths on each input terminate at the same time by entering its unique halting state after writing 0 (rejection) or 1 (acceptance) in the start cell of the designated output tape (see [17] for the discussion on the timing problem). Thus, the length of a computation path on input $x$ is regarded as the running time of the QTM on $x$. The transition function $\delta$ of a QTM can be seen as an operator (called a time-evolution operator) that transforms a superposition of configurations at time $t$ to another superposition of configurations at time $t+1$. A QTM is called well-formed if its time-evolution operator is unitary. Moreover, a QTM is said to have $\tilde{\mathbb{C}}$-amplitudes if all amplitudes in $\delta$ are drawn from set $\tilde{\mathbb{C}}$, where $\tilde{\mathbb{C}}$ is the set of all complex numbers whose real and imaginary parts are approximated deterministically to within $2^{-n}$ in time polynomial in $n$. For a well-formed QTM $M$ and an input $|\phi\rangle$, the notation $\operatorname{Prob}_{M}[M(|\phi\rangle)=1]$ denotes the acceptance probability of $M$ on input $|\phi\rangle$. Similarly, $\operatorname{Prob}_{M}[M(|\phi\rangle)=0]$ denotes the rejection probability of $M$ on $|\phi\rangle$.

\subsection{From Classical Inputs to Quantum Inputs}

We have used classical (binary) strings as standard inputs given into quantum computations. As a result, any quantum complexity class, such as NQP or BQP [4], is defined to be a collection of subsets of $\Sigma^{*}$. Since a QTM acts as a unitary operator, it is legitimate to feed the QTM with a quantum state as an input. We call such an input a quantum input for clarity. As in the definition of $\mathrm{QMA}(k)$, for instance, such quantum inputs play an essential role. We thus need to expand a set of strings to a set of qustrings by considering a qustring as an input given to an underlying QTM. We use the following notation. For each $m, n \in \mathbb{N}^{+}$, let $\Phi_{n}^{m}$ denote the collection of all $m$-tuples $\left(\left|\phi_{1}\right\rangle,\left|\phi_{2}\right\rangle, \ldots,\left|\phi_{m}\right\rangle\right)$ such that each $\left|\phi_{i}\right\rangle$ is a qustring of size $n$. Such an $m$-tuple is expressed as $|\vec{\phi}\rangle$ and also seen as a tensor product $\left|\phi_{1}\right\rangle\left|\phi_{2}\right\rangle \cdots\left|\phi_{m}\right\rangle$ when the size of each $\left|\phi_{i}\right\rangle$ is known. For brevity, the notation $\ell(|\vec{\phi}\rangle)$ means the sum $\sum_{i=1}^{m} \ell\left(\left|\phi_{i}\right\rangle\right)$. We also set $\Phi_{\infty}^{m}=\bigcup_{n \geq 1} \Phi_{n}^{m}$ and $\Phi_{\infty}^{*}=\bigcup_{m \geq 1} \Phi_{\infty}^{m}$. 
The introduction of quantum inputs gives rise to an important issue, which is not present in the classical framework: the duplication of an input. The repetition of a quantum computation on a classical input is seen in, e.g., the proof of $B Q P^{B Q P}=B Q P$ [3]. Nevertheless, the situation may change when we deal with a quantum input. Since a fundamental principle of quantum computation, the so-called no-cloning theorem, interdicts the duplication of an arbitrary quantum input, we cannot redo even the same quantum computation on a single quantum input unless the copies of the quantum input are given a priori. To establish a coherent but concise theory of quantum computation over $\Phi_{\infty}^{*}$, we need to allow the underlying quantum computation to access the quantum input repeatedly without disturbing other quantum states. Schematically, we supply a sufficient number of its copies as "auxiliary inputs." This guarantees the quantum extension of many existing complexity classes, such as BQP, to enjoy the same structural properties.

For later convenience, we first expand the function class \#QP [18], which originally consists of certain quantum functions mapping from $\Sigma^{*}$ to the unit real interval $[0,1]$. The notation ${ }^{*} \# Q P$ is given in this paper to denote the corresponding extension-the collection of quantum functions mapping from $\Phi_{\infty}^{*}$ to $[0,1]$. Since $\Phi_{\infty}^{*}$ is a continuous space, these quantum functions are inherently continuous. For simplicity, write $|\vec{\phi}\rangle^{\otimes k}$ for $k$ copies of $|\vec{\phi}\rangle$, which can be viewed as a tensor product of $k$ identical $|\vec{\phi}\rangle$ 's (as long as the size of $|\vec{\phi}\rangle$ is known).

Definition 1 A function $f$ from $\Phi_{\infty}^{*}$ to $[0,1]$ is in *\#QP if there exist a polynomial $q$ and a polynomial-time, $\tilde{\mathbb{C}}$-amplitude, well-formed $Q T M M$ such that, for every $m \in \mathbb{N}^{+}$and every $|\vec{\phi}\rangle \in \Phi_{\infty}^{m}, f(|\vec{\phi}\rangle)=\operatorname{Prob}_{M}\left[M\left(|\vec{\phi}\rangle^{\otimes q(\ell(|\vec{\phi}\rangle))}\right)=1\right]$.

We reserve the standard notation \#QP to denote the class of quantum functions whose domains are $\Sigma^{*}$ (i.e., those functions are obtained from Definition 1 by replacing $|\vec{\phi}\rangle$ with $x$ from $\Sigma^{*}$ ).

To distinguish a set of qustrings from a set of classical strings, we use the terminology, a quantum set, for a set $A \subseteq \Phi_{\infty}^{*}$. A collection of quantum sets is called a quantum complexity class (which conventionally refers to any classical class related to quantum computations). From a different perspective, a classical set can be viewed as a "projection" of its corresponding quantum set. For a quantum set $A \subseteq \Phi_{\infty}^{*}$, its classical part $\check{A}$ is given as follows:

$$
\check{A}=\left\{\left\langle s_{1}, s_{2}, \ldots, s_{m}\right\rangle\left|m \in \mathbb{N}^{+}, s_{1}, \ldots, s_{m} \in \Sigma^{*},\left(\left|s_{1}\right\rangle,\left|s_{2}\right\rangle, \ldots,\left|s_{m}\right\rangle\right) \in A\right\},\right.
$$

where \langle\rangle is an appropriate pairing function from $\bigcup_{m \geq 1}\left(\Sigma^{*}\right)^{m}$ to $\Sigma^{*}$. Thus, any quantum class $\mathcal{C}$ naturally induces its classical part $\{\check{A} \mid A \in \mathcal{C}\}$. In a similar way, \#QP is also viewed as the "projection" of *\#QP.

A relativized version of *\#QP is defined by substituting oracle QTMs for non-oracle QTMs in Definition 1, where an oracle QTM can make a query of the form $|x\rangle|b\rangle\left(x \in \Sigma^{*}\right.$ and $\left.b \in\{0,1\}\right)$ by which oracle $A$ transforms $|x\rangle|b\rangle$ into $(-1)^{b \cdot A(x)}|x\rangle|b\rangle$ in a single step. 


\subsection{From Decision Problems to Partial Decision Problems}

We described in the previous subsection how to expand classical sets to quantum sets. The next step might be to expand well-known complexity classes, such as NQP and BQP, to classes of quantum sets. Unfortunately, since $\Phi_{\infty}$ is a continuous space, we cannot expand all classical classes in this way (for example, BQP). One of the resolutions is to consider "partial" decision problems. (See, e.g., [5] for classical partial decision problems.) In this paper, we define a partial decision problem to be a pair $(A, B)$ such that $A, B \subseteq \Phi_{\infty}^{*}$ and $A \cap B=\varnothing$, where $A$ indicates a set of accepted qustrings and $B$ indicates a set of rejected qustrings. The legal region of $(A, B)$ is $A \cup B$. For consistency with classical decision problems, we should refer $A$ to as $(A, \bar{A})$, where $\bar{A}=\Phi_{\infty}^{*}-A$, and call it a total decision problem. The notions of inclusion, union, and complement are introduced in the following manner: let $(A, B)$ and $(C, D)$ be any partial decision problems and let $E$ be the intersection of their legal regions; that is, $(A \cup B) \cap(C \cup D)$.

1. Inclusion: $(A, B) \subseteq(C, D)$ iff $A \subseteq C$ and $A \cup B=C \cup D$.

2. Intersection: $(A, B) \cap(C, D) \stackrel{\text { def }}{=}(A \cap C,(B \cup D) \cap E)$.

3. Union: $(A, B) \cup(C, D) \stackrel{\text { def }}{=}((A \cup C) \cap E, B \cap D)$.

4. Complementation: $\overline{(A, B)} \stackrel{\text { def }}{=}(B, A)$.

Now, we focus on classes of partial decision problems. To denote such a class, we use the special notation ${ }^{*} \mathcal{C}$, whose asterisk signifies the deviation from total decision problems. The partial classical part of a partial decision problem $(A, B)$ is $(\check{A}, \check{B})$. When $\check{A} \cup \check{B}=\Sigma^{*}$, we call $(\check{A}, \check{B})$ the total classical part of $(A, B)$ and simply write $\check{A}$ instead of $(\check{A}, \check{B})$ as before. Notationally, let $\mathcal{C}$ denote the collection of total classical parts in ${ }^{*} \mathcal{C}$. We call $\mathcal{C}$ the total classical part of ${ }^{*} \mathcal{C}$.

For later use, we expand BQP to the class of partial decision problems. In a similar fashion, we can expand other classes, such as NQP and PQP [18].

Definition 2 Let $a, b$ be any two functions from $\mathbb{N}$ to $[0,1]$ such that $a(n)+$ $b(n)=1$ for all $n \in \mathbb{N}$. A partial decision problem $(A, B)$ is in ${ }^{*} \operatorname{BQP}(a, b)$ if there exists a quantum function $f \in{ }^{*} \# \mathrm{QP}$ such that, for every $|\vec{\phi}\rangle \in \Phi_{\infty}^{*}$, (i) if $|\vec{\phi}\rangle \in A$ then $f(|\vec{\phi}\rangle) \geq a(\ell(|\vec{\phi}\rangle))$ and (ii) if $|\vec{\phi}\rangle \in B$ then $f(|\vec{\phi}\rangle) \leq b(\ell(|\vec{\phi}\rangle))$. For simplicity, write ${ }^{*} \mathrm{BQP}$ for ${ }^{*} \mathrm{BQP}(3 / 4,1 / 4)$.

It is important to note that the total classical part of ${ }^{*} \mathrm{BQP}$ coincides with the standard definition of BQP, e.g., given in [4]. Since the duplication of a quantum input is available for free of charge, we can perform a standard majority-vote algorithm for a set in ${ }^{*} \mathrm{BQP}$ to amplify its success probability. Therefore, we obtain ${ }^{*} \mathrm{BQP}={ }^{*} \mathrm{BQP}\left(1-2^{-p(n)}, 2^{-p(n)}\right)$ for any polynomial $p$. 


\section{The $\exists^{\mathrm{Q}}$-Operator and the $\forall^{\mathrm{Q}}$-Operator}

The process of defining a new complexity class $\mathcal{D}$ from a basis class $\mathcal{C}$ can be naturally viewed as an application of an operator, which maps $\mathcal{C}$ to $\mathcal{D}$. As seen in Section 1, the $\exists$-operator over classical sets is an abstraction of nondeterministic computation (as in NP $=\exists \cdot \mathrm{P}$ ) and its complement is called the $\forall$-operator. First, we generalize these operators to the ones whose scopes are classes of partial decision problems.

Definition 3 Let ${ }^{*} \mathcal{C}$ be any quantum complexity class of partial decision problems. A partial decision problem $(A, B)$ is in ${ }^{*} \exists \cdot{ }^{*} \mathcal{C}$ if there exist a polynomial $p$ and a partial decision problem $(C, D)$ in ${ }^{*} \mathcal{C}$ such that, for all vectors $|\vec{\phi}\rangle \in \Phi_{\infty}^{*}$,

i) if $|\vec{\phi}\rangle \in A$ then $\exists x \in \Sigma^{p(\ell(|\vec{\phi}\rangle))}[(|x\rangle,|\vec{\phi}\rangle) \in C]$ and

ii) if $|\vec{\phi}\rangle \in B$ then $\forall x \in \Sigma^{p(\ell(|\vec{\phi}\rangle))}[(|x\rangle,|\vec{\phi}\rangle) \in D]$.

The class ${ }^{*} \forall \cdot{ }^{*} \mathcal{C}$ is defined similarly by exchanging the roles of the quantifiers in conditions $i$ ) and ii). In accordance to the standard notation, $\exists \cdot{ }^{*} \mathcal{C}$ and $\forall \cdot{ }^{*} \mathcal{C}$ denote the total classical parts of ${ }^{*} \exists \cdot{ }^{*} \mathcal{C}$ and ${ }^{*} \forall \cdot{ }^{*} \mathcal{C}$, respectively.

The class QMA uses a quantum quantifier, whose scope is qustrings of polynomial size instead of classical strings of polynomial length. Generalizing such a quantum quantifier, we introduce a quantum analogue of the $\exists$ - and $\forall$-operators as follows. Our approach is quite different from that of Green et al. [8], who defined the N-operator as an abstraction of NQP.

Definition 4 Let ${ }^{*} \mathcal{C}$ be a quantum complexity class of partial decision problems. A partial decision problem $(A, B)$ is in ${ }^{*} \exists Q \cdot{ }^{*} \mathcal{C}$ if there exist a polynomial $p$ and a partial decision problem $(C, D) \in{ }^{*} \mathcal{C}$ such that, for every $|\vec{\phi}\rangle \in \Phi_{\infty}^{*}$,

i) if $|\vec{\phi}\rangle \in A$ then $\exists|\psi\rangle \in \Phi_{p(\ell(|\vec{\phi}\rangle))}[(|\psi\rangle,|\vec{\phi}\rangle) \in C]$ and

ii) if $|\vec{\phi}\rangle \in B$ then $\forall|\psi\rangle \in \Phi_{p(\ell(|\vec{\phi}\rangle))}[(|\psi\rangle,|\vec{\phi}\rangle) \in D]$.

Similarly, the class ${ }^{*} \mathrm{Q} .{ }^{*} \mathcal{C}$ is defined by exchanging the roles of quantifiers in conditions i) and ii) above. The notations $\exists^{\mathrm{Q}} \cdot{ }^{*} \mathcal{C}$ and $\forall^{\mathrm{Q}} \cdot{ }^{*} \mathcal{C}$ denote the total classical parts of ${ }^{*} \exists^{Q} .{ }^{*} \mathcal{C}$ and ${ }^{*} \forall^{Q} .{ }^{*} \mathcal{C}$, respectively. More generally, write ${ }^{*} \exists_{1}^{\mathrm{Q}} \cdot{ }^{*} \mathcal{C}$ for ${ }^{*} \exists^{\mathrm{Q}} \cdot{ }^{*} \mathcal{C}$ and recursively define ${ }^{*} \exists_{m+1}^{\mathrm{Q}} \cdot{ }^{*} \mathcal{C}$ as ${ }^{*} \exists^{\mathrm{Q}} \cdot\left({ }^{*} \exists_{m}^{\mathrm{Q}} \cdot{ }^{*} \mathcal{C}\right)$. Similarly, ${ }^{*} \forall_{m+1}^{\mathrm{Q}} \cdot{ }^{*} \mathrm{C}$ is defined.

Obviously, if ${ }^{*} \mathcal{C} \subseteq{ }^{*} \mathcal{D}$ then $\exists^{Q} .{ }^{*} \mathcal{C} \subseteq \exists^{Q}$. ${ }^{*} \mathcal{D}$ and ${ }^{*} \exists^{Q} .{ }^{*} \mathcal{C} \subseteq{ }^{*} \exists^{Q} .{ }^{*} \mathcal{D}$.

We next show that the $\exists^{Q}$ - and $\forall^{Q}$-operators indeed expand the classical $\exists$ and $\forall$-operators, respectively. Proving this claim, however, requires underlying class ${ }^{*} \mathcal{C}$ to satisfy a certain condition, which is given in the following definition.

Definition 5 1. A quantum set $B \subseteq \Phi_{\infty}^{*}$ is called classically separable if the following condition holds: for every $m, n \in \mathbb{N}^{+}$and every $|\vec{\phi}\rangle \in \Phi_{n}^{m}$, if either $\langle\vec{x} \mid \vec{\phi}\rangle=0$ or $(|\vec{x}\rangle,|\vec{\psi}\rangle) \in B$ for all $\vec{x} \in\left(\Sigma^{n}\right)^{m}$, then $(|\vec{\phi}\rangle,|\vec{\psi}\rangle) \in B$. 
2. A quantum complexity class ${ }^{*} \mathcal{C}$ of partial decision problems is said to be classically simulatable if, for every partial decision problem $(A, B) \in{ }^{*} \mathcal{C}$, there exist a partial decision problem $(C, D) \in{ }^{*} \mathcal{C}$ such that (i) $C$ and $D$ are classically separable and (ii) for all $m, n \in \mathbb{N}^{+}$and all $\vec{x} \in\left(\Sigma^{n}\right)^{m},(|\vec{x}\rangle,|\vec{\psi}\rangle) \in A$ $\Longleftrightarrow(|\vec{x}\rangle,|\vec{\psi}\rangle) \in C$ and $(|\vec{x}\rangle,|\vec{\psi}\rangle) \in B \Longleftrightarrow(|\vec{x}\rangle,|\vec{\psi}\rangle) \in D$.

The above notion stems from the proof of QMA containing NP. The classes of partial decision problems dealt with in this paper are indeed classically simulatable.

Lemma 6 If a quantum complexity class ${ }^{*} \mathcal{C}$ of partial decision problems is classically simulatable, then ${ }^{*} \exists \cdot{ }^{*} \mathcal{C} \subseteq{ }^{*} \exists^{\mathrm{Q}} \cdot{ }^{*} \mathcal{C}$ and ${ }^{*} \forall \cdot{ }^{*} \mathcal{C} \subseteq{ }^{*} \forall \mathrm{Q} \cdot{ }^{*} \mathcal{C}$.

The proof of the first claim of Lemma 6 easily follows from the definition of classical-simulatability. The second claim comes from the fact that if ${ }^{*} \mathcal{C}$ is classically simulatable then so is $\operatorname{co}^{*} \mathcal{C}$, where $\operatorname{co-}^{*} \mathcal{C}$ denotes the collection of partial decision problems whose complements belong to ${ }^{*} \mathcal{C}$.

\section{The Quantum Polynomial Hierarchy}

The classical $\exists$ - and $\forall$-operators are useful tools to expand complexity classes. In the early 1970s, Meyer and Stockmeyer [13] introduced the polynomial hierarchy, whose components are obtained from $\mathrm{P}$ with alternating applications of these operators; namely, $\Sigma_{k+1}^{\mathrm{P}}=\exists \cdot \Pi_{k}^{\mathrm{P}}$ and $\Pi_{k+1}^{\mathrm{P}}=\forall \cdot \Sigma_{k}^{\mathrm{P}}$ for each level $k \in \mathbb{N}^{+}$. The polynomial hierarchy has continued to be a center of research in complexity theory. The introduction of the $\exists^{Q}$ - and $\forall^{Q_{-}}$-operators enables us to consider a quantum analogue of the polynomial hierarchy and explore its structural properties in light of the strength of quantum computability. We call this new hierarchy the quantum polynomial hierarchy (QP-hierarchy, for short). The basis of the QP hierarchy is *BQP opposed to $\mathrm{P}$ since two-sided bounded-error computations are more realistic in the quantum setting. Each level of the QP hierarchy is obtained from its lower level by a finite number of applications of the same quantum operator (either $\exists^{Q_{-}}$or $\forall^{Q_{-}}$operators). Although any repetition of the same classical operator has no significance in the polynomial hierarchy, as Kobayashi et al. [12] pointed out, there might be a potentially essential difference between a single quantum quantifier and multiple quantum quantifiers of the same type. The precise definition of the QP hierarchy is given as follows.

Definition 7 Let $a, b$ be two functions from $\mathbb{N}$ to $[0,1]$ such that $a(n)+b(n)=1$ for all $n \in \mathbb{N}$. Let $k \in \mathbb{N}$ and $m \in \mathbb{N}^{+}$. The quantum polynomial hierarchy (QP hierarchy, for short) constitutes the following complexity classes of partial decision problems.
i) ${ }^{*} \Sigma_{0, m}^{\mathrm{QP}}(a, b)={ }^{*} \Pi_{0, m}^{\mathrm{QP}}(a, b)={ }^{*} \mathrm{BQP}(a, b)$.
ii) ${ }^{*} \Sigma_{k+1, m}^{\mathrm{QP}}(a, b)={ }^{*} \exists_{m}^{\mathrm{Q}} \cdot{ }^{*} \Pi_{k, m}^{\mathrm{QP}}(a, b)$.
iii) ${ }^{*} \Pi_{k+1, m}^{\mathrm{QP}}(a, b)={ }^{*} \forall_{m}^{\mathrm{Q}} \cdot{ }^{*} \Sigma_{k, m}^{\mathrm{QP}}(a, b)$. 
Let ${ }^{*} \Sigma_{k}^{\mathrm{QP}}(a, b)=\bigcup_{m \geq 1}{ }^{*} \Sigma_{k, m}^{\mathrm{QP}}(a, b)$ and ${ }^{*} \Pi_{k}^{\mathrm{QP}}(a, b)=\bigcup_{m \geq 1}{ }^{*} \Pi_{k, m}^{\mathrm{QP}}(a, b)$. Furthermore, let ${ }^{*} \mathrm{QPH} \mathrm{H}_{m}(a, b)=\bigcup_{k>0}\left({ }^{*} \Sigma_{k, m}^{\mathrm{QP}}(a, b) \cup^{*} \Pi_{k, m}^{\mathrm{QP}}(a, b)\right)$ and ${ }^{*} \mathrm{QPH}(a, b)=$ $\bigcup_{m>1}{ }^{*} \mathrm{QPH}_{m}(a, b)$. Their total classical parts are denoted (without asterisks) $\Sigma_{k}^{\mathrm{QP}}(a, b), \Pi_{k}^{\mathrm{QP}}(a, b)$, and $\mathrm{QPH}(a, b)$.

For brevity, we write ${ }^{*} \Sigma_{k}^{\mathrm{QP}}$ for ${ }^{*} \Sigma_{k}^{\mathrm{QP}}(3 / 4,1 / 4),{ }^{*} \Pi_{k}^{\mathrm{QP}}$ for ${ }^{*} \Pi_{k}^{\mathrm{QP}}(3 / 4,1 / 4)$, and ${ }^{*} \mathrm{QPH}$ for ${ }^{*} \mathrm{QPH}(3 / 4,1 / 4)$. Likewise, we can define their total classical parts $\Sigma_{k}^{\mathrm{QP}}, \Pi_{k}^{\mathrm{QP}}$, and QPH. The choice of the value $(3 / 4,1 / 3)$ is artificial; however, a standard majority-vote algorithm can amplify $(3 / 4,1 / 4)$ to $(1-$ $\left.2^{-p(n)}, 2^{-p(n)}\right)$ for an arbitrary polynomial $p$. Due to our general framework, it is likely that $\Sigma_{1}^{Q P}$ is strictly larger than $\bigcup_{k>1} \mathrm{QMA}(k)$. From this reason, $\Sigma_{1}^{Q P}$ can be regarded as Quantum NP, as discussed in Section 1.

Several alternative definitions of the QP hierarchy are possible. Here, we present three alternatives. The first one uses the function class ${ }^{*} \mathrm{Q} o p t \# \Sigma_{k, m}^{\mathrm{QP}}-\mathrm{a}$ generalization of Qopt\#QP in [19]-introduced as follows: a quantum function $f$ from $\Phi_{\infty}^{*}$ to $[0,1]$ is in ${ }^{*} Q o p t \# \Sigma_{k, m}^{Q P}$ if there exist a polynomial $p$ and a quantum function $g \in{ }^{*} \# \mathrm{QP}$ such that, for every $|\vec{\phi}\rangle \in \Phi_{\infty}^{*}$,

$$
f(|\vec{\phi}\rangle)=\sup _{\left|\vec{\psi}_{1}\right\rangle\left|\vec{\psi}_{2}\right\rangle} \cdots \operatorname{opr}_{\left|\vec{\psi}_{k}\right\rangle}^{(k)}\left\{g\left(|\vec{\phi}\rangle,\left|\vec{\psi}_{1}\right\rangle,\left|\vec{\psi}_{2}\right\rangle, \ldots,\left|\vec{\psi}_{k}\right\rangle\right)\right\}
$$

where opr ${ }^{(k)}=\sup$ if $k$ is odd and opr ${ }^{(k)}=\inf$ otherwise, and each $\left|\vec{\psi}_{i}\right\rangle$ is an $m$-tuple $\left(\left|\psi_{i, 1}\right\rangle,\left|\psi_{i, 2}\right\rangle, \ldots,\left|\psi_{i, m}\right\rangle\right)$ with each $\left|\psi_{i, j}\right\rangle$ running over all qustrings of size $p(\ell(|\vec{\phi}\rangle))$. The class ${ }^{*} Q$ opt\# $\Sigma_{k, m}^{Q P}$ gives a succinct way to define the $k$ th level of the QP hierarchy.

Lemma 8 Let $k, m \geq 1$ and let $a, b$ be any two functions from $\mathbb{N}$ to $[0,1]$ satisfying $a(n)+b(n)=1$ for all $n \in \mathbb{N}$. A partial decision problem $(A, B)$ is in ${ }^{*} \Sigma_{k, m}^{\mathrm{QP}}(a, b)$ iff there exists a quantum function $f$ in ${ }^{*}$ Qopt $\# \Sigma_{k, m}^{\mathrm{QP}}$ such that, for every $|\vec{\phi}\rangle \in \Phi_{\infty}^{*}$, (i) if $|\vec{\phi}\rangle \in A$ then $f(|\vec{\phi}\rangle) \geq a(\ell(|\vec{\phi}\rangle)$ ) and (ii) if $|\vec{\phi}\rangle \in B$ then $f(|\vec{\phi}\rangle) \leq b(\ell(|\vec{\phi}\rangle))$.

In the second alternative definition, we use vectors whose components are described by classical strings. For each $n, r \in \mathbb{N}^{+}$, denote by $\tilde{\Phi}_{n}(r)$ the collection of all vectors $|\phi\rangle$ (not necessarily elements in a Hilbert space) such that $|\phi\rangle$ has the form $\sum_{s:|s|=n} \alpha_{s}|s\rangle$, where each complex number $\alpha_{s}$ is expressed as a pair of two binary fractions of $r$ bits. If $r \geq \log (1 / \epsilon)+n+2$ for $\epsilon>0$, such $|\phi\rangle$ satisfies $\left.\left|\sum_{s:|s|=n}\right| \alpha_{s}\right|^{2}-1 \mid \leq \epsilon$. Note that any element in $\tilde{\Phi}_{n}$ can be expressed as a binary string of length $r 2^{n+1}$ (since each $\alpha_{s}$ needs $2 r$ bits and we have exactly $2^{n}$ such $\alpha_{s}$ 's). Thus, the cardinality of $\tilde{\Phi}_{n}$ is $2^{r 2^{n+1}}$. For our purpose, we allow each *\#QP-function to take any vector in $\tilde{\Phi}_{n}(r)$ as its input.

Lemma 9 Let $k, m \in \mathbb{N}^{+}$. A partial decision problem $(A, B)$ is in ${ }^{*} \Sigma_{k, m}^{Q P}$ iff there exist a polynomial $p$ and a quantum function $f \in{ }^{*} \# \mathrm{QP}$ such that, for every series of qustrings $|\vec{\phi}\rangle$ in $\Phi_{\infty}^{*}$, 
i) if $|\vec{\phi}\rangle \in A$ then $\exists\left|\overrightarrow{\xi_{1}}\right\rangle \forall\left|\overrightarrow{\xi_{2}}\right\rangle \cdots Q_{k}\left|\overrightarrow{\xi_{k}}\right\rangle\left[f\left(|\vec{\phi}\rangle,\left|\overrightarrow{\xi_{1}}\right\rangle,\left|\overrightarrow{\xi_{2}}\right\rangle, \ldots,\left|\overrightarrow{\xi_{k}}\right\rangle\right) \geq 3 / 4\right]$ and

ii) if $|\vec{\phi}\rangle \in B$ then $\left.\forall\left|\overrightarrow{\xi_{1}}\right\rangle|\exists| \overrightarrow{\xi_{2}}\right\rangle \cdots \bar{Q}_{k}\left|\overrightarrow{\xi_{k}}\right\rangle\left[f\left(|\vec{\phi}\rangle,\left|\overrightarrow{\xi_{1}}\right\rangle,\left|\overrightarrow{\xi_{2}}\right\rangle, \ldots,\left|\overrightarrow{\xi_{k}}\right\rangle\right) \leq 1 / 4\right]$,

where each variable $\left|\vec{\xi}_{i}\right\rangle$ runs over all series of $m$ vectors in $\tilde{\Phi}_{p(\ell(\mid \vec{\phi})))}(3 p(\ell(|\vec{\phi}\rangle))), Q_{k}=\forall$ if $k$ is even and $Q_{k}=\exists$ otherwise, and $\bar{Q}_{k}$ is the opposite quantifier of $Q_{k}$.

The last alternative definition is much more involved and we need extra notions and notation. Firstly, we give a method of translating a qustring $|\phi\rangle$ into a series of unitary matrices that generate $|\phi\rangle$. Let $\mathbb{C}$ be the set of all complex numbers, $I$ the $2 \times 2$ identity matrix, and $\lambda$ the empty string. Fix $n \in \mathbb{N}$ and $|\phi\rangle \in \Phi_{n+1}$ and assume that $|\phi\rangle=\sum_{s:|s|=n+1} \gamma_{s}|s\rangle$, where each $\gamma_{s}$ is in $\mathbb{C}$. For each $s \in \Sigma \leq n$, set $g_{s}=\sqrt{\sum_{t}\left|\gamma_{s 0 t}\right|^{2}+\sum_{t}\left|\gamma_{s 1 t}\right|^{2}}$ and define a $2 \times 2$ matrix $U^{(s)}$ as follows: let $U^{(s)}|b\rangle=\left(\sqrt{\sum_{t}\left|\gamma_{s 0 t}\right|^{2}} / g_{s}\right)|0\rangle+(-1)^{b}\left(\sqrt{\sum_{t}\left|\gamma_{s 1 t}\right|^{2}} / g_{s}\right)|1\rangle$ if $|s|\left\langle n\right.$; otherwise, let $\left.U^{(s)} \mid b\right\rangle=\left(\gamma_{s 0} / g_{s}\right)|0\rangle+(-1)^{b}\left(\gamma_{s 1} / g_{s}\right)|1\rangle$. The series $\mathcal{U}=$ $\left\langle U^{(s)} \mid s \in \Sigma^{\leq n}\right\rangle$ is called the generator of $|\phi\rangle$ since $|\phi\rangle=U_{n} U_{n-1} \cdots U_{0}\left|0^{n+1}\right\rangle$, where $U_{0}=U^{(\lambda)} \otimes I^{\otimes n}$ and $U_{k}=\sum_{s:|s|=k}|s\rangle\langle s| \otimes U^{(s)} \otimes I^{\otimes n-k}$ for each $k$, $1 \leq k \leq n$.

Secondly, we consider a good approximation of a given generator. For any $2 \times 2$ matrix $U=\left(u_{i j}\right)_{1 \leq i, j \leq 2}$ on $\mathbb{C}$ and any $\epsilon>0, \tilde{U}=\left(\tilde{u}_{i j}\right)_{1 \leq i, j \leq 2}$ is called the $\epsilon$-fragment of $U$ if each $\tilde{u}_{i j}$ represents the first $\lceil\log (1 / \epsilon)\rceil$ bits of the infinite binary fractions of the real and imaginary parts of $u_{i j}$ (so that $\left|u_{i j}-\tilde{u}_{i j}\right| \leq 2 \epsilon$ ). In this case, $\tilde{U}$ satisfies $\|U-\tilde{U}\| \leq 4 \epsilon$. If $\tilde{U}^{(s)}$ is the $\epsilon$-fragment of $U^{(s)}$ for all $s \in \Sigma^{\leq n}$, the series $\tilde{\mathcal{U}} \stackrel{\text { def }}{=}\left\langle\tilde{U}^{(s)} \mid s \in \Sigma^{\leq n}\right\rangle$ is also called the $\epsilon$-fragment of $\mathcal{U}$. We assume a natural encoding scheme of $\tilde{\mathcal{U}}$ into oracle $\langle\tilde{\mathcal{U}}\rangle$ so that $\tilde{\mathcal{U}}$ can be retrieved by $O\left(2^{n} \log (1 / \epsilon)\right)$ queries to oracle $\langle\tilde{\mathcal{U}}\rangle$.

Lemma 10 There exists a well-formed QTM $M_{0}$ that satisfies the following condition: for every $\epsilon>0$, every $n \in \mathbb{N}$, and every generator $\mathcal{U}$ of a qustring $|\phi\rangle \in \Phi_{n+1}$, if $\tilde{\mathcal{U}}$ is the $\epsilon 2^{-n-4}$-fragment of $\mathcal{U}$, then $M_{0}$ with oracle $\langle\tilde{\mathcal{U}}\rangle$ halts on input $\left|0^{n+1}\right\rangle$ in time polynomial in $1 / \epsilon$ and $n$ and satisfies $\||\phi\rangle\langle\phi|-\rho \|_{\mathrm{tr}} \leq \epsilon$, where $\rho$ is the density matrix obtained from the final superposition of $M_{0}$ by tracing out all but the output-tape content and $\|A\|_{\operatorname{tr}}$ denotes the trace of $\sqrt{A^{\dagger} A}$.

Proof Sketch. The desired $M_{0}$ works as follows: at step 0 , write $\left|0^{n+1}\right\rangle$ in the work tape. Let $s 0^{n-k+1}$ be the string written in the work tape after step $k-1$. At step $k$, make appropriate queries to oracle $\langle\tilde{\mathcal{U}}\rangle$ to realize quantum gate $G^{(s)}$ that simulates $\tilde{U}^{(s)}$ with accuracy at most $\delta$ (i.e., $\left\|G^{(s)}-\tilde{U}^{(s)}\right\| \leq \delta$ ). Then, apply $|s\rangle\langle s| \otimes G^{(s)} \otimes I^{\otimes n-k}$ (or $G^{(\lambda)} \otimes I^{\otimes n}$ if $s=\lambda$ ) to $\left|s 0^{n-k+1}\right\rangle$.

Finally, the third alternative definition of the QP hierarchy is given in Lemma 11. A merit of Lemma 11 is no need of the duplication of quantum inputs given to underlying QTMs. Note that Lemma 11 can be further generalized to nongenerators. 
Lemma 11 Let $k \geq 1$. For any classical set $A \subseteq \Sigma^{*}, A$ is in $\Sigma_{k, 1}^{\mathrm{QP}}$ iff there exist two polynomials $p, q$ and a polynomial-time well-formed oracle QTM M such that, for all $x \in \Sigma^{*}$,

i) if $x \in A$ then $\exists \mathcal{U}_{1} \forall \mathcal{U}_{2} \cdots Q_{k} \mathcal{U}_{k}\left[\operatorname{Prob}_{M}\left[M^{\left\langle\tilde{u}_{1}, \tilde{\mathcal{U}}_{2}, \ldots, \tilde{u}_{k}\right\rangle}(x)=1\right] \geq 3 / 4\right]$ and

ii) if $x \notin A$ then $\forall \mathcal{U}_{1} \exists \mathcal{U}_{2} \cdots \bar{Q}_{k} \mathcal{U}_{k}\left[\operatorname{Prob}_{M}\left[M^{\left\langle\bar{u}_{1}, \tilde{U}_{2}, \ldots, \tilde{u}_{k}\right\rangle}(x)=1\right] \leq 1 / 4\right]$, where $Q_{k}=\forall$ if $k$ is even and $Q_{k}=\exists$ otherwise, $\bar{Q}_{k}$ is the opposite quantifier of $Q_{k}$, each variable $\mathcal{U}_{i}$ runs over all generators of qustrings of size $p(|x|)$, each $\tilde{\mathcal{U}}_{i}$ is the $2^{-q(|x|)}$-fragment of $\mathcal{U}_{i}$, and $M$ on input $x$ behaves as follows: whenever it makes a query, it writes $\left|1^{i}\right\rangle$ in an query tape and runs $M_{0}$ (defined in Lemma 10) on input $\left|0^{p(|x|)}\right\rangle$ with oracle $\left\langle\tilde{\mathcal{U}}_{i}\right\rangle$.

\section{Fundamental Properties of the QP Hierarchy}

Although the QP hierarchy looks more complex than its classical counterpart, the QP hierarchy shares many fundamental properties with the polynomial hierarchy. In the next proposition, we present without proofs a short list of fundamental properties of the QP hierarchy.

Proposition 12 1. For every $k \in \mathbb{N}$, co- ${ }^{*} \Sigma_{k}^{\mathrm{QP}}={ }^{*} \Pi_{k}^{\mathrm{QP}}$ and $\operatorname{co-}{ }^{*} \Pi_{k}^{\mathrm{QP}}=$ ${ }^{*} \Sigma_{k}^{\mathrm{QP}}$.

2. For each $k \in \mathbb{N},{ }^{*} \Sigma_{k}^{\mathrm{QP}}$ and ${ }^{*} \Pi_{k}^{\mathrm{QP}}$ are closed under intersection and union.

3. For each $k \in \mathbb{N},{ }^{*} \Sigma_{k}^{\mathrm{QP}} \cup^{*} \Pi_{k}^{\mathrm{QP}} \subseteq{ }^{*} \Sigma_{k+1}^{\mathrm{QP}} \cap^{*} \Pi_{k+1}^{\mathrm{QP}}$.

4. For every $k \in \mathbb{N}^{+}, \bigcup_{m>0}{ }^{*} \exists_{m}^{\mathrm{Q}} \cdot\left({ }^{*} \Sigma_{k}^{\mathrm{QP}} \cap{ }^{*} \Pi_{k}^{\mathrm{QP}}\right)={ }^{*} \Sigma_{k}^{\mathrm{QP}}$.

5. Let $k \in \mathbb{N}^{+}$. If ${ }^{*} \Sigma_{k}^{\mathrm{QP}}={ }^{*} \Pi_{k}^{\mathrm{QP}}$ then ${ }^{*} \Sigma_{k}^{\mathrm{QP}}={ }^{*} \mathrm{QPH}$.

6. For each $k \in \mathbb{N}^{+},{ }^{*} \exists \cdot{ }^{*} \Sigma_{k}^{\mathrm{QP}}={ }^{*} \exists^{\mathrm{Q}} \cdot{ }^{*} \Sigma_{k}^{\mathrm{QP}}={ }^{*} \Sigma_{k}^{\mathrm{QP}}$.

Of the above items, item 6 is specifically meant for the QP hierarchy and requires the classical-simulatability of the QP hierarchy, which is shown below.

Lemma 13 For each $k \in \mathbb{N},{ }^{*} \Sigma_{k}^{\mathrm{QP}}$ and ${ }^{*} \Pi_{k}^{\mathrm{QP}}$ are classically simulatable.

Proof. Since the base case $k=0$ is easy, we skip this case and prove the general case $k>0$. Let $m \in \mathbb{N}^{+}$and $(A, B)$ be any partial decision problem in ${ }^{*} \Sigma_{k, m}^{Q P}$. There exists a function $f \in{ }^{*}$ Qopt\# $\Sigma_{k, m}^{Q P}$ that satisfies Lemma 8 for $(A, B)$. Assume that $f$ has the form

$$
f(|\vec{\phi}\rangle,|\vec{\psi}\rangle)=\sup _{\left|\vec{\xi}_{1}\right\rangle\left|\vec{\xi}_{2}\right\rangle} \cdots \operatorname{opr}_{\left|\vec{\xi}_{k}\right\rangle}^{(k)}\left\{h\left(\left|\vec{\xi}_{1}\right\rangle,\left|\vec{\xi}_{2}\right\rangle, \ldots,\left|\vec{\xi}_{k}\right\rangle,|\vec{\phi}\rangle,|\vec{\psi}\rangle\right)\right\}
$$

for a certain quantum function $h$ in ${ }^{*} \#$ QP. For brevity, write $|\Xi\rangle$ for $\left(\left|\vec{\xi}_{1}\right\rangle, \ldots,\left|\vec{\xi}_{k}\right\rangle\right)$. Letting $h^{\prime}(|\Xi\rangle,|\vec{\phi}\rangle,|\vec{\psi}\rangle)=\sum_{\vec{x}}|\langle\vec{x} \mid \vec{\phi}\rangle|^{2} h(|\Xi\rangle,|\vec{x}\rangle,|\vec{\psi}\rangle)$, we define $g$ as $g(|\vec{\phi}\rangle,|\vec{\psi}\rangle)=\sup _{\left|\vec{\xi}_{1}\right\rangle} \inf _{\left|\vec{\xi}_{2}\right\rangle} \cdots \operatorname{opr}_{\left|\vec{\xi}_{k}\right\rangle}^{(k)}\left\{h^{\prime}\left(\left|\vec{\xi}_{1}\right\rangle,\left|\vec{\xi}_{2}\right\rangle, \ldots,\left|\vec{\xi}_{k}\right\rangle,|\vec{\phi}\rangle,|\vec{\psi}\rangle\right)\right\}$. Note 
that $g(|\vec{x}\rangle,|\vec{\psi}\rangle) \leq g(|\vec{\phi}\rangle,|\vec{\psi}\rangle)$ if $\langle\vec{x} \mid \vec{\phi}\rangle \neq 0$, since $h^{\prime}$ satisfies $h^{\prime}(|\Xi\rangle,|\vec{x}\rangle,|\vec{\psi}\rangle) \leq$ $h^{\prime}(|\Xi\rangle,|\vec{\phi}\rangle,|\vec{\psi}\rangle)$ for any $|\Xi\rangle$. It follows that, for every $\vec{x}, g(|\vec{x}\rangle,|\vec{\psi}\rangle)=f(|\vec{x}\rangle,|\vec{\psi}\rangle)$.

To complete the proof, defining $C=\{(|\vec{\phi}\rangle,|\vec{\psi}\rangle)|g(|\vec{\phi}\rangle,|\vec{\psi}\rangle) \geq 3 / 4\}$ and $D=\{(|\vec{\phi}\rangle,|\vec{\psi}\rangle)|g(|\vec{\phi}\rangle,|\vec{\psi}\rangle) \leq 1 / 4\}$, we show that $C$ and $D$ are classically separable. Let $|\vec{\phi}\rangle$ be fixed arbitrarily. Assume that $\forall \vec{x}[\langle\vec{x} \mid \vec{\phi}\rangle=0 \vee(|\vec{x}\rangle,|\vec{\psi}\rangle) \in$ $C]$. We want to show that $(|\vec{\phi}\rangle,|\vec{\psi}\rangle) \in C$. Assume otherwise. We then have $g(|\vec{\phi}\rangle,|\vec{\psi}\rangle)<3 / 4$. Take any element $\vec{x}$ such that $\langle\vec{x} \mid \vec{\phi}\rangle \neq 0$. It follows that $g(|\vec{x}\rangle,|\vec{\psi}\rangle) \leq g(|\vec{\phi}\rangle,|\vec{\psi}\rangle)<3 / 4$, which implies $(|\vec{x}\rangle,|\vec{\psi}\rangle) \notin C$, a contradiction. The case for $D$ is similar. Therefore, ${ }^{*} \Sigma_{k}^{\mathrm{QP}}$ is classically simulatable.

Next, we give rudimentary but meritorious upper and lower bounds of the QP hierarchy. The exponential hierarchy consists of the following classes: $\Delta_{0}^{\mathrm{EXP}}=\Sigma_{0}^{\mathrm{EXP}}=\Pi_{0}^{\mathrm{EXP}}=\operatorname{EXP}\left(=\operatorname{DTIME}\left(2^{n^{O(1)}}\right)\right), \Delta_{k}^{\mathrm{EXP}}=\operatorname{EXP}^{\Sigma_{k}^{\mathrm{P}}}$, $\Sigma_{k}^{\mathrm{EXP}}=\mathrm{NEXP}^{\Sigma_{k-1}^{\mathrm{P}}}$, and $\Pi_{k}^{\mathrm{EXP}}=\operatorname{co-} \Sigma_{k}^{\mathrm{EXP}}$ for every $k \in \mathbb{N}^{+}$. Let EXPH denote the union of $\Sigma_{k}^{\mathrm{EXP}}$ for all $k \in \mathbb{N}$. We show that $\mathrm{PH} \subseteq \mathrm{QPH} \subseteq \mathrm{EXPH}$.

Theorem 14 For each $k>0, \Sigma_{k}^{\mathrm{P}} \subseteq \Sigma_{k}^{\mathrm{QP}} \subseteq \Sigma_{k}^{\mathrm{EXP}}$. Thus, PH $\subseteq \mathrm{QPH} \subseteq$ EXPH.

Theorem 14 yields the following collapse: if $\Pi_{k}^{\mathrm{P}} \subseteq \Sigma_{k}^{\mathrm{QP}}$ then $\mathrm{PH} \subseteq \Sigma_{k}^{\mathrm{QP}}$.

Proof of Theorem 14. We show the first inclusion that $\Sigma_{k}^{\mathrm{P}} \subseteq \Sigma_{k}^{\mathrm{QP}}$. The proof is done by induction on $k \geq 0$. The base case $k=0$ follows from $\mathrm{P} \subseteq \mathrm{BQP}$. Let $k>0$. By the induction hypothesis, it follows that $\Sigma_{k-1}^{\mathrm{P}} \subseteq \Sigma_{k-1}^{\mathrm{QP}}$, which further implies $\Pi_{k-1}^{\mathrm{P}} \subseteq \Pi_{k-1}^{\mathrm{QP}}$. Since ${ }^{*} \Pi_{k-1}^{\mathrm{QP}}$ is classically simulatable (Lemma 13), Lemma 6 yields $\exists \cdot{ }^{*} \Pi_{k-1}^{\mathrm{QP}} \subseteq \exists^{\mathrm{Q}} \cdot{ }^{*} \Pi_{k-1}^{\mathrm{QP}}$. Thus,

$$
\Sigma_{k}^{\mathrm{P}}=\exists \cdot \Pi_{k-1}^{\mathrm{P}} \subseteq \exists \cdot{ }^{*} \Pi_{k-1}^{\mathrm{QP}} \subseteq \bigcup_{m>0} \exists_{m}^{\mathrm{Q}} \cdot{ }^{*} \Pi_{k-1}^{\mathrm{QP}}=\Sigma_{k}^{\mathrm{QP}} .
$$

The second inclusion $\Sigma_{k}^{\mathrm{QP}} \subseteq \Sigma_{k}^{\mathrm{EXP}}$ follows from Lemma 9. Let $m \in \mathbb{N}^{+}$ and let $A$ be any set in $\Sigma_{k, m}^{\mathrm{QP}}$. Take a polynomial $p$ and a quantum function $f \in{ }^{*} \# \mathrm{QP}$ guaranteed by Lemma 9 for $(A, \bar{A})$. We construct an alternating TM $N$ as follows: on input $x$, start with an $\exists$-state, generate $k$ vectors $\left|\overrightarrow{\xi_{1}}\right\rangle,\left|\overrightarrow{\xi_{2}}\right\rangle, \ldots,\left|\overrightarrow{\xi_{k}}\right\rangle$ in $\left(\tilde{\Phi}_{p(|x|)}(3 p(|x|))\right)^{m}$ by alternately entering $\forall$ - and $\exists$-states, and check if $f\left(|x\rangle,\left|\overrightarrow{\xi_{1}}\right\rangle,\left|\overrightarrow{\xi_{2}}\right\rangle, \ldots,\left|\overrightarrow{\xi_{k}}\right\rangle\right) \geq 3 / 4$. This last check is done in exponential time since $f$ runs in time polynomial in $|x|$. Since any exponential-time alternating TM with $k$-alternation starting with the $\exists$-state is known to characterize $\Sigma_{k}^{\mathrm{EXP}}, A$ belongs to $\Sigma_{k}^{\mathrm{EXP}}$.

In the end of this section, we discuss the issue of complete problems. Each level of the polynomial hierarchy is known to have complete problems. Unfortunately, it is believed that classes like BQP and QMA lack such complete 
problems because of their acceptance criteria. Dealing with partial decision problems, however, allows us to go around this difficulty. (See, e.g., [5] for the NP-completeness for classical partial decision problems.) With an appropriate modification of classical completeness proofs, we can show that each level of the QP hierarchy indeed has a "complete" partial decision problem (under a deterministic reduction). An important open problem is to find natural complete partial decision problems for each level of the QP hierarchy.

\section{Relativized QP Hierarchies}

We have introduced a quantum analogue of the polynomial hierarchy and explored its basic properties and its relationship to the polynomial hierarchy. In this last section, we give simple relativized results related to the $\mathrm{QP}$ hierarchy. The relativized $Q P$ hierarchy relative to oracle $A,\left\{\Sigma_{k}^{\mathrm{QP}}(A), \Pi_{k}^{\mathrm{QP}}(A) \mid k \in \mathbb{N}\right\}$, is obtained simply by changing the basis class ${ }^{*} \# \mathrm{QP}$ to its relativized version ${ }^{*} \# \mathrm{QP}^{A}$.

Proposition 15 1. There exists a recursive oracle $A$ such that $\mathrm{P}^{A}=\mathrm{PH}^{A}=$ $\mathrm{QPH}^{A}$.

2. There exists a recursive oracle $B$ such that $\Sigma_{0}^{\mathrm{QP}}(B) \neq \Sigma_{1}^{\mathrm{QP}}(B) \neq$ $\Sigma_{2}^{\mathrm{QP}}(B)$.

3. There exists a recursive oracle $C$ such that $\Sigma_{k}^{\mathrm{P}}(C) \neq \Sigma_{k}^{\mathrm{QP}}(C)$ for all $k \in \mathbb{N}^{+}$.

For the first claim of Proposition 15, it suffices to construct $A$ such that $\mathrm{P}^{A}=\Sigma_{1}^{\mathrm{QP}}(A)$ since this yields $\Sigma_{1}^{\mathrm{QP}}(A)=\Pi_{1}^{\mathrm{QP}}(A)$, which further implies $\Sigma_{1}^{\mathrm{QP}}(A)=\mathrm{QPH}^{A}$. The $\operatorname{desired~set~} A$ is built by stages: at each stage, pick one relativized \#QP-function $f$ and encode its outcome into one string that cannot be queried by $f$. This is possible because an oracle QTM that witnesses $f$ runs in polynomial time.

The second claim of Proposition 15 follows from a generalization of the result co-UP ${ }^{B} \nsubseteq \bigcup_{k>1} \mathrm{QMA}(k)^{B}$ [12]. In fact, we can show by modifying Ko's argument [11] a slightly stronger result: there exists an oracle $B$ satisfying that co-UP ${ }^{B} \nsubseteq \Sigma_{1}^{\mathrm{QP}}(B)$ and $\mathrm{BP} \cdot \Sigma_{1}^{\mathrm{QP}}(B)=\Sigma_{1}^{\mathrm{QP}}(B)$ with Schöning's BPoperator. It is easy to see that this yields the desired claim. Of particular interest is to show that co-UP ${ }^{B} \nsubseteq \Sigma_{1}^{Q P}(B)$. This is done by cultivating a lower bound technique of a certain type of a real-valued circuit. Since the acceptance probability of an oracle QTM compatation can be expressed by a multilinear polynomial of small degree [2], we can convert a $\Sigma_{1, m}^{\mathrm{QP}}(A)$-computation into a family of circuits $C$ of depth-2 (that work on real numbers) such that (i) the top gate of $C$ is a $M A X$-gate of fanin at most $m 2^{n 2^{n+1}}$ and (ii) all bottom gates of $C$ are polynomial-gates of degree at most $n$ with fanin $2^{n}$, where a $M A X$-gate is a gate that takes real numbers as its inputs and outputs their maximal value and a polynomial-gate of degree $k$ refers to a gate that computes a multilinear polynomial of degree exactly $k$. The fanin of such a polynomial gate is the number of variables actually appearing in its underlying polynomial. 
The existence of $B$ comes from the fact that such a family of circuits cannot approximate to within $1 / 3$ any Boolean function of large block sensitivity (e.g., a co-UP ${ }^{B}$-computation). The oracle separation at higher levels of the QP hierarchy is one of the remaining open problems.

The third claim of Proposition 15 follows from the result $\mathrm{QMA}^{C} \nsubseteq \mathrm{MA}^{C}$ [16] and its generalization. The detail will appear in the complete version of this extended abstract.

\section{References}

[1] L. M. Adleman, J. DeMarrais, and M. A. Huang. Quantum computability, SIAM J. Comput. 26 (1997), 1524-1540.

[2] R. Beals, H. Buhrman, R. Cleve, M. Mosca, and R. de Wolf. Quantum lower bounds by polynomials, in Proceedings of the 39th Annual Symposium on Foundations of Computer Science, pp.352-361, 1998.

[3] C. H. Bennett, E. Bernstein, G. Brassard, and U. Vazirani. Strengths and weaknesses of quantum computing, SIAM J. Comput. 26 (1997), 1510-1523.

[4] E. Bernstein and U. Vazirani. Quantum complexity theory, SIAM J. Comput. 26 (1997), 1411-1473.

[5] D. Du and K. Ko. Theory of Computational Complexity, John Wiley \& Sons, Inc., 2000.

[6] S. Fenner, F. Green, S. Homer, and R. Pruim. Determining acceptance probability for a quantum computation is hard for the polynomial hierarchy, Proceedings of the Royal Society of London, Ser.A, 455 (1999), 3953-3966.

[7] L. Fortnow and J. Rogers. Complexity limitations on quantum computation, $J$. Comput. System Sci. 59 (1999), 240-252.

[8] F. Green, S. Homer, C. Moore, and C. Pollett. Counting, fanout, and the complexity of quantum ACC, Quantum Information and Computation, 2 (2002), $35-65$.

[9] A. Kitaev. "Quantum NP", Public Talk at AQIP'99: the 2nd Workshop on Algorithms in Quantum Information Processing, DePaul University, 1999.

[10] E. Knill. Quantum randomness and nondeterminism, Technical Report LAUR96-2186, 1996. See also LANL quant-ph/9610012.

[11] K. Ko. Separating and collapsing results on the relativized probabilistic polynomial-time hierarchy, J. ACM 37 (1990), 415-438.

[12] H. Kobayashi, K. Matsumoto, and T. Yamakami. Quantum Merlin Arthur proof systems, manuscript, 2001. See also LANL quant-ph/0110006.

[13] A. R. Meyer and L. J. Stockmeyer. The equivalence problem for regular expressions with squaring requires exponential time, in Proceedings of the 13th Annual Symposium on Switching and Automata Theory, pp.125-129, 1972.

[14] U. Schöning. Probabilistic complexity classes and lowness, J. Comput. System and Sci. 39 (1989), 84-100.

[15] K. Wagner. The complexity of combinatorial problems with succinct input representation, Acta Inf. 23 (1986), 325-356. 
[16] J. Watrous. Succinct quantum proofs for properties of finite groups, in Proceedings of the 41st Annual Symposium on Foundations of Computer Science, pp.537-546, 2000.

[17] T. Yamakami. A foundation of programming a multi-tape quantum Turing machine, in Proceedings of the 24th International Symposium on Mathematical Foundation of Computer Science, Lecture Notes in Computer Science, Vol.1672, pp.430-441, 1999.

[18] T. Yamakami. Analysis of quantum functions, in Proceedings of the 19th International Conference on Foundations of Software Technology and Theoretical Computer Science, Lecture Notes in Computer Science, Vol.1738, pp.407-419, 1999.

[19] T. Yamakami. Quantum optimization problems, manuscript, 2002. See LANL quant-ph/0204010.

[20] T. Yamakami and A. C. Yao. $\mathrm{NQP}_{\mathbf{C}}=$ co-C=P, Inf. Process. Let. 71 (1999), 63-69. 